
8 9

\section{Vigilance performance during acute exercise}

\author{
Francisco González-Fernández ${ }^{1,2,4}$, Jennifer L. Etnier ${ }^{3}$, Mikel Zabala ${ }^{4}$, \& Daniel Sanabria ${ }^{1,2}$
}

1. Mind, Brain \& Behavior Research Center, Universidad de Granada, Spain.

2. Departamento de Psicología Experimental, Universidad de Granada, Spain.

3. Department of Kinesiology, University of North Carolina at Greensboro, USA.

4. Departamento de Educación Física y Deportiva, Universidad de Granada, Granada, Spain.

Corresponding author:

Daniel Sanabria,daniel@ugr.es

Running head: Vigilance during exercise

Published in the International Journal of Sport Psychology

doi: 10.7352/IJSP 2017.48.000

Raw data from this study can be downloaded here

https://zenodo.org/record/7431584\#.Y5g57uLMIqt

Acknowledgments

This study was supported by grants from the Junta de Andalucía, Spain (Proyecto de Excelencia, SEJ-6414) to Daniel Sanabria and from the Ministerio de Economía y Competitividad, Spain (PSI2013-46385) to Daniel Sanabria and Jennifer Etnier and from the Ministerio de Economía y 7 Competitividad, Spain (PSI2016-75956-P) to Daniel Sanabria and Mikel Zabala. 
Abstract

Vigilance, the cognitive function that determines goal maintenance and attention

deployment, is involved in many day life activities, which often implicate physical activity. We investigated vigilance performance during exercise, with a particular focus on exercise intensity. In Experiment 1, participants performed the psychomotor vigilance task (PVT) for 5' at 40\%, 60\%, $80 \%$ and $100 \%$ of the ventilatory anaerobic threshold (VAT), in different sessions. The results showed that PVT performance depended on exercise intensity with an "optimal" point at $80 \%$ of VAT. In Experiment 2, participants completed a 45' version of the PVT at a low-effort (control) condition and at a 75\% VAT light-moderate effort condition. Reaction times were faster at the lightmoderate effort than at the low-effort condition over the 45'. The present study demonstrated that

43 the vigilance performance changes during acute exercise, an effect that is moderated by effort intensity.

Keywords: Physical activity; sustained attention; reaction time; cognition; psychomotor vigilance task. 
Introduction

Vigilance determines goal maintenance and the deployment of attention in order to respond appropriately (quickly and accurately) to relevant stimuli (Sarter et al., 2001). Vigilance is involved in numerous everyday life activities, many of them including physical activity. In effect, human behaviours occur more often in movement than in the motionless, non-physically stressful conditions of the majority of cognitive neuroscience laboratories (see Walsh 2014, for discussion on this issue). Many investigators have taken this into consideration, resulting in a rapid growth of the interest on the links between brain/cognition and sport/exercise in the last two decades. However, to the best of our knowledge, there are no previous studies investigating vigilance during exercise.

A review of the literature reveals that performance in cognitive tasks that range from simple reaction time (RT) tasks to more attention demanding (e.g., working memory) tasks is affected by concurrent physical exercise (Chang, Labban, Gapin \& Etnier, 2012). These effects have been linked to variations in activation/arousal (e.g., Davranche \& Audiffren, 2004) induced by physiological changes (e.g., blood lactate, plasma catecholamines, cortical activation) at central and peripheral levels (McMorris \& Hale, 2015). The direction and magnitude of these physiological changes depend on the intensity of the exercise, a moderating variable influencing cognitive performance during acute exercise (Brisswalter, et al., 2002). Indeed, improvements in cognitive performance have been most commonly associated with light-to-moderate intensity exercise, while lower or higher intensities (as a function of the participant's anaerobic threshold) result in performance decline or in no significant variations (with respect to the peak performance; e.g., Chmura et al., 1998). Even though there is evidence of the influence of acute exercise during cognitive performance, little is known regarding the effect on vigilance as noted earlier. In this first empirical study of the effects of exercise intensity on vigilance, we take an exploratory approach (cf. Wagenmakers, Wetzels, Borsboom, Van der Maas, \& Kievit, 2012) to the 
study of vigilance during exercise. Participants were asked to perform a modified version of the psychomotor vigilance task (PVT) while pedaling on an indoor ergometer. The emphasis on vigilance in the PVT is created by the highly unpredictable target onset (see Methods for details) that requires participants to maintain their attention focused on the task and to re-orient attention to task goals in case of mind wandering and distraction, in order to respond as rapidly as possible to the target onset. Performance in the PVT has been shown to depend on the overall attention state of the participant, that can be influenced by various factors such as sleep deprivation (e.g., Basner \& Dinges, 2011), circadian rhythmicity (e.g., Correa, Molina, \& Sanabria, 2013; Muto et al., 2016) or cardiovascular fitness (e.g., Luque-Casado, Perakakis, Ciria, \& Sanabria, 2016a; Luque-Casado et al., 2016b). The demands of attention of the PVT are also supported by neuroimaging studies (e.g., Drummond et al. 2005) showing brain activity typically associated with the sustained attention network (cf. Lawrence, Ross, Hoffmann, Garavan, \& Stein, 2003) and the allocation of attentional resources (cf. Culham, Cavanagh, \& Kanwisher, 2001). Luque-Casado et al. (2016a, 2016b) also showed the involvement of top-down attention in the PVT using event-related brain and cardiac potentials. We believe, on the basis of this prior research, that the PVT is far more than a simple visuo-motor reaction time (RT) task as it involves attention demands that tap the sustained attention (or tonic alerting in terms of Posner's attention network systems; see Petersen \& Posner, 2012, for a review) brain network that has been identified in numerous neuroimaging studies (including the midcingulate cortex, prefrontal cortex, ventral premotor cortex, temporoparietal junction, among other areas; Rosenberg et al., 2016; see Langner \& Eickhoff, 2013, for a review).

In sum, the current empirical study was conceived to investigate vigilance during exercise, considering exercise intensity as a crucial moderator. In Experiment 1, participants performed the PVT during 5 ' bouts of exercise at 40\%, 60\%, 80\% and 100\% of Ventilatory Anaerobic Threshold (VAT) on different days. In Experiment 2, participants performed a 45' PVT during low-effort and light-moderate effort corresponding to $75 \%$ of the VAT. On the basis of the previous research on cognitive task performance during physical exercise, we expected that exercising at light-to- 
moderate intensity (between $60 \%$ and $80 \%$ of VAT) would improve RT performance in the PVT with respect to lower and higher intensities.

\section{General Methods}

\section{Participants}

All participants were recruited via flyers and they reported normal or corrected to normal vision, had no history of neurological of physical disorders, and gave informed consent prior to the start of the experiment (see Table 1). They participated in the studies in exchange for $\$ 30$ or extra course credits. This research was approved by the institutional review board of the University of Granada.

\section{Equipment and materials}

Insert Table 1 around here

A RS800CX Polar monitor (Polar Electro, Finland) was used to monitor and record heart rate (HR) during the exercise sessions. Ventilatory gas exchange was measured during a submaximal incremental exercise test using a Jaeger Master Screen gas analyzer and a Viasprint $150 \mathrm{P}$ cycle-ergometer. In addition to HR recordings, the rating of perceived exertion (RPE) scale (Borg, 1998) was used to measure perceived effort immediately after the experimental sessions. The RPE scale ranged from 6 (not exertion) to 20 (maximal exertion).

A LCD 15.6"' laptop was used to present the stimuli for the PVT. The center of the screen was situated $60-80 \mathrm{~cm}$ from the participant's head and at eye level. The E-Prime software (Psychology Software Tools, Pittsburgh, PA, USA) was used to control stimulus presentation and data collection. A wireless button connected to the computer USB-2 port was used to collect responses.

Psychomotor vigilance task 

centre of the screen. After a random interval between 2000 and $10000 \mathrm{~ms}$, the circle started to fill in with red beginning from the vertical radius and proceeding in a clockwise direction at an angular velocity of 0.094 degrees per second. Verbal and written instructions were given to the participant prior to the start of the PVT in every session, stressing that they had to fixate on the centre of the screen, try not to move their eyes, and respond as quickly as possible (while avoiding anticipation errors) as soon as the circle started filling in. Feedback in ms was given during a $300 \mathrm{~ms}$ inter-trial interval. Then, the next trial began.

\section{Procedure}

The participant visited the lab on five occasions in Experiment 1 and three in Experiment 2, always at the same time of day, between 9:30 am - 5:00 pm, and separated by at least 48 hours and no more than 96 hours. The Polar HR monitor was attached around the chest of the participant upon arrival at the laboratory. During the first session, participants performed a sub-maximal incremental fitness test on a stationary ergometer. They were equipped with a facemask connected to the metabolic cart. We followed the guidelines of the American College of Sports Medicine (ACSM, 2010) to ensure the safety of the participants. The protocol (Astrand, 1965) consisted of a submaximal incremental test with a fixed cadence of 60 revolutions per min (rpm). The warm-up started at $0 \mathrm{~W}$ and the workload was increased by $10 \mathrm{~W}$ every min until min 5 . The participant began the exercise phase pedaling at $50 \mathrm{~W}$, and the workload was increased $25 \mathrm{~W}$ every two min for women and $50 \mathrm{~W}$ every two min for men. After each increase, the workload remained stable for the next 2 minutes. Determination of VAT was based on the respiratory gas-exchange method $(\mathrm{RER})\left([\mathrm{RER}]=\mathrm{CO}_{2}\right.$ production $/ \mathrm{O}_{2}$ consumption $)$, which detected the VAT at the point at which RER exceeds the cut-off value of 1.0 (Myers \& Ashley, 1997; Yeh et al., 1995). The researcher knew that the participant had reached the VAT when the RER was equal to 1.00 and did not drop below that level during the 2 min constant load period or during the next load step increase in wattage, never reaching the 1.1 RER. The submaximal test ended once the VAT was reached. VAT was measured in $\mathrm{ml}^{*} \mathrm{~kg}^{-1} * \mathrm{~min}^{-1}$, and the results of this test were used to establish the power load in 
the subsequent experimental conditions. After completing the submaximal test and having approximately 5 minutes to recover, participants completed 8-10 trials of the PVT while pedaling to familiarize them with the task.

In Experiment 1, participants completed 4 experimental sessions on separate days and in a counterbalanced order. On each session, after a 3' warm up, participants performed the PVT for 5' while pedaling either at $40 \%$ of the VAT, $60 \%$ of the VAT, $80 \%$ of the VAT or $100 \%$ of the VAT. The participant's HR was monitored throughout the effort sessions to ensure the maintenance of the proper intensity and reduced or increased the cyclo-ergometer resistance in steps of $1 \mathrm{~W}$ as necessary.

In Experiment 2, participants completed the PVT, after a 3' warm up, during a 45' bout of low-effort and a $45^{\prime}$ bout of light-moderate effort corresponding to $75 \%$ of the VAT, on separate days. The order of presentation of the two effort conditions was counterbalanced.

Design, data reduction, and statistical analysis

Experiment 1 consisted of a one-way within-participants design with the factor of effort condition $(40 \%, 60 \%, 80 \%, 100 \%$ of the VAT $)$. Participants completed $47.46( \pm 2.55), 48.38( \pm$ $2.61), 48.17( \pm 2.39), 46.83( \pm 2.18)$ trials in the $40 \%, 60 \%, 80 \%, 100 \%$ of the VAT effort conditions, respectively.

Experiment 2 consisted of the within-participants factors of effort condition (light-moderateeffort and low-effort) and time-on-task (9 blocks of 5' each). Note that the $45^{\prime}$ ' were divided in blocks of 5' to investigate the time course of RT in the PVT. Participants completed $430.94( \pm 9.42)$ and $432.94( \pm 10.68)$ trials in the light-moderate-effort and low-effort conditions, respectively.

Trials with RTs below 100 ms (1.39\% for Experiment 1, and 0.87\%, for Experiment 2) were assumed to represent anticipation errors and were discarded from the analysis (Luque-Casado et al., 2016, a,b). Analyses of variance (ANOVAs) were used to analyze the RT, HR and RPE data.

Statistically significant effects were further analyzed by paired-sample t-tests corrected by HolmBonferroni for multiple comparisons. Effect size is indicated with Cohen's d for t-tests and partial 
eta squared for Fs. The Greenhouse-Geisser correction was applied when sphericity was violated (Jennings and Wood, 1976). If so, corrected probability values are reported.

\section{Results}

\section{Experiment 1}

A repeated measures ANOVA with participants' mean RPE data (see Table 2), revealed a significant main effect of effort condition, $F(3,69)=496,48, p<.001, \eta^{2}$ partial $=.95$. T-tests showed significant differences between all conditions, all $p \mathrm{~s}<.001$. A repeated measures ANOVA with participants' mean HR data from Experiment 1 (see Table 2), revealed a significant main effect of effort condition, $F(3,69)=6852,50, p<.001, \eta^{2}$ partial $=.99$. Paired-sample t-tests showed significant differences between all conditions, all $p \mathbf{s}<.001$.

\section{Insert Table 2 around here}

A repeated measures ANOVA with participants' mean RT, revealed a significant main effect of effort condition $F(1.44,33.17)=7.87, p<.01, \eta^{2}$ partial $=.25$ (see Figure 1). RTs followed a quadratic trend, $F(1,23)=19.00, p<.001$, with peak performance in $80 \%$ VAT effort condition $(321 \pm 24.72$ $\mathrm{ms})$ and the worst performance in the $100 \%$ VAT effort condition $(370 \pm 74.40 \mathrm{~ms})$. Pairwise comparisons showed significant differences in RT between the $80 \%$ VAT effort condition and the $100 \%$ VAT effort condition, $t(23)=-3.61, p<.008, \mathrm{~d}=-0.89$, and between the $60 \%$ VAT effort condition and the $100 \%$ VAT effort condition, $t(23)=-3.25, p<.017, \mathrm{~d}=-0.71$. The comparison between $60 \%$ VAT effort condition and $80 \%$ VAT, $t(23)=2.67, p<.054, \mathrm{~d}=0.42$, and the comparison between $40 \%$ VAT effort condition and $80 \%$ VAT effort condition, $t(23)=2.41, p<.072, \mathrm{~d}=0.56$, failed to reach statistical significance. Comparison between the $40 \%$ VAT effort condition and the $60 \%$ VAT effort condition, and comparison between the $40 \%$ VAT effort condition and 100\% VAT effort condition were not significant, $t(23)=1.08, p<.10, \mathrm{~d}=0.25$ and $t(23)=-2.02, p<.28, \mathrm{~d}=-0.54$, respectively. 
The peak performance in Experiment 1 was obtained in the 5' version of the PVT at the $80 \%$

VAT. In Experiment 2, we decided to use a slightly lower intensity, corresponding to the 75\% VAT instead, to investigate the time course of the "optimal" effort condition on vigilance over a substantially longer exercise duration. That way, we ensured a constant effort during the longer 45' bout. In fact, the 75\% VAT intensity in Experiment 2 resulted in the same RPE as the $80 \%$ VAT intensity in Experiment 1. Moreover, the difference in the relative effort intensities $(80 \% \mathrm{VAT}$ and $75 \%$ VAT) was only $9 \mathrm{bpm}$, around $3 \%$ of $\mathrm{HR}_{\max }$ (see Table 2 ).

\section{Experiment 2}

A t-test with the data from the RPE scale and HR showed higher values in the lightmoderate effort condition than in the low-effort condition, in both cases, $t(18)=3,55, p<.001$, $\mathrm{d}=1.90$, and $t(18)=11,41, p<.001, \mathrm{~d}=3.82$, respectively (see Table 2 ).

A repeated measures ANOVA with participants' mean RT revealed a significant main effect of effort condition, $F(1,17)=5,42, p<.03, \eta^{2}$ partial $=.24$, with shorter RTs in the light-moderate-effort condition than in the low-effort condition. The analysis revealed a significant main effect of timeon-task, $F(3.03,51.62)=17,42, p<.001, \eta^{2}$ partial $=.50$, with RT increasing over the course of the task. The interaction between effort condition and time-on-task, $F<1$, was not significant (see Figure 2).

Insert Figure 2 around here

At this point, we wondered whether the relative effect on PVT performance (with respect to the low-effort condition) of the 75\% VAT bout of effort in Experiment 2 was significantly different from that in the $80 \%$ VAT condition in Experiment 1 . If so, one would expect a statistically significant interaction between experiment $(1,2)$ and effort condition (low-effort, light-moderateeffort). We selected the data from the $40 \%$ VAT condition as the low-effort condition in Experiment 1. Data from the first 5' of each effort session in Experiment 2 were entered in the analysis. 
$246 \eta_{\text {partial }}^{2}=.21$, with shorter RTs in Experiment 1 than in Experiment 2. The analysis also showed a 247 significant main effect of effort condition, $F(1,40)=8,03, p<.01, \eta^{2}$ partial $=.16$, with shorter RTs in 248 light-moderate-effort than in the low-effort condition. Crucially, the interaction between experiment 249 and effort condition was not significant, $F<1$, i.e., the magnitude of the main effect of effort condition 250 did not significantly differ as a function of experiment.

\section{Discussion}

The present study confirmed that the participants' state of vigilance changed during exercise and that it was moderated by the intensity of the physical activity. The results in Experiment 1 showed a peak RT performance at $80 \%$ VAT intensity in the PVT. The results of Experiment 2 revealed a significant main effect of effort condition with shorter RTs in the $75 \%$ VAT lightmoderate intensity exercise condition than in the low-effort condition. The main effect of time-ontask was also significant, with RT gradually increasing over the course of the task. The null interaction in Experiment 2, together with the main effect of effort condition, suggests that, although task performance suffered from a similar time-on-task decrement in both conditions, exercising at light-moderate intensity resulted in a constant RT enhancement. Note that the results in Experiment 2 appear to contradict previous outcomes reported when studies are summarized meta-analytically suggesting that at least $20 \mathrm{~min}$ of exercise are needed to observe enhancement of cognitive performance (Chang, Labban, Gapin \& Etnier, 2012).

The outcome of the present study confirmed that the participants' state of vigilance changes during exercise (compared to a low-effort condition) and that it is moderated by the intensity of the physical activity. Crucially, exercising at light-moderate effort intensity resulted in constant vigilance enhancement during a relatively long session of effort, at least for $45^{\prime}$. These findings extend previous research on the cognition-during-exercise topic to the case of vigilance or sustained attention. Importantly, all effort conditions were matched in terms of dual-task demands. Therefore, 
any variation in vigilance performance was presumably due to the physiological changes induced by the particular effort condition.

Exercising elicits a variety of physiological alterations (e.g., increases in core temperature, cortical blood flow, heart rate, catecholamine concentration; McMorris \& Hale, 2015). Many of these physiological responses have been linked to enhanced activation/arousal (that relates to the overall activation/excitability of cortical neurons; Langner \& Eickhoff, 2013; Oken, Salinsky, \& Elsas, 2006) that may in turn be responsible for the observed variations in PVT performance as a function of exercise intensity. Interestingly, the peak vigilance performance was found at lightmoderate effort intensity. On one hand, this latter result is consistent with recent accounts suggesting that activation of the reticular system during moderate exercise would boost cognitive performance (Dietrich \& Audiffren, 2011; McMorris \& Hale 2012). Indeed, there is a wealth of literature supporting the notion that performance in the PVT is dependent upon the overall activation state of the participant when influenced by sleep deprivation (Dorrian, Rogers, \& Dinges, 2005) or time of day (Correa et al., 2014). On the other hand, exercising at light-moderate intensity might have also enhanced attention control, as suggested by previous research (McMorris et al., 2009; Pesce, Capranica, Tesittore, \& Figura, 2002). Thus, given the demands of focused attention of the PVT, it would also be reasonable to state that exercising at light-moderate intensity improved performance by enhancing attention control.

While the $80 \%$ VAT bout of effort seemed to enhance PVT performance in Experiment 1, the largest RTs were found in the 100\% VAT exercise condition. That detrimental effect of exercise on cognitive performance in the PVT was similar to that observed in previous studies that have measured performance in tasks with high demands of attention during high intense exercise (see Brisswalter, et al., 2002; Chang, Labban, Gapin \& Etnier, 2012; Lambourne \& Tomporowski, 2010, for reviews). Increasing exercise intensity up to the $100 \%$ VAT might have simply resulted in surpassing the "optimal" arousal level for the PVT in line with the Yerkes-Dodson law (Yerkes \& Dodson, 1908) which suggests that performance improves with increasing arousal up to a "moderate" level and then shows detriments with additional increases in arousal. Alternatively, it 
299 might have also induced a higher competition between the PVT and the cycling task (which 300 demanded participants to keep the effort intensity and the pedaling rate constant throughout the $5^{\prime}$ ) 301 resulting in a detrimental effect in the PVT (see Dietrich \& Audiffren, 2011; Pontifex \& Hillman, 3022007 for a related account). In any case, according to the current results, the "optimal" vigilance 303 performance during exercise is achieved at an intensity corresponding to the $75-80 \%$ of the VAT 304 and future research will be necessary to elucidate the underlying mechanisms that explain this 305 effect.

306 In sum, the present empirical study demonstrated that the vigilance state during a task with 307 high demands of vigilance attention is affected during acute exercise, an effect that is moderated by 308 effort intensity. It also contributes to the extant research on cognitive performance during exercise 309 and opens interesting avenues for future research. 


\section{References}

American College of Sports Medicine (2010). ACSM's guidelines for exercise testing and prescription. 9th. ed. London: Lippincott Williams \& Wilkins.

Astrand, P.O. (1965). Work tests with the bicycle ergometer. Varberg, Sweden: AB Cykelfabriken Monark.

Basner M., \& Dinges D.F., (2011). Maximizing sensitivity of the psychomotor vigilance test (PVT) to sleep loss. Sleep, 34(5), 581-591.

Borg, G. (1998). Borg's perceived exertion and pain scales. Champaign, IL, US: Human Kinetics.

Brisswalter, J., Collardeau, M., \& Rene, A. (2002). Effects of acute physical exercise characteristics on cognitive performance. Sports Medicine, 32, 555-566.

Chang, Y.K., Labban, J.D., Gapin, J.I., \& Etnier J.L. (2012). The effects of acute exercise on cognitive performance: A meta-analysis. Brain Research, 1453, 87-101.

Chmura, J., Krysztofiak, H., Ziemba, A.W. (1998). Psychomotor performance during prolonged exercise above and below the blood lactate threshold. European Journal of Applied Physiology, 77, 77-80

Correa, A., Molina, E., Sanabria, D. (2014). Effects of chronotype and time of day on the vigilance decrement during simulated driving. Accident Analysis \& Prevention, 67, 113-118

Culham, J. C., Cavanagh, P., \& Kanwisher, N. G. (2001). Attention response functions: characterizing brain areas using fMRI activation during parametric variations of attentional load. Neuron, 32(4), 737-745.

Davranche, K., \& Audiffren M. (2004). Facilitating effects of exercise on information processing. Journal of Sports Sciences, 22, 419-428.

Dietrich A., \& Audiffren. M. (2011). The reticular-activating hypofrontality (RAH) model of acute exercise. Neuroscience \& Biobehavioral Reviews. 35(6), 1305-1325.

Dorrian, J., Rogers, N.L., \& Dinges, D.F. (2005). Psychomotor vigilance performance: Neurocognitive assay sensitive to sleep loss. In Kushida, C. A. (Ed.), Sleep 
Marcel Dekker.

339 Drummond, S.P., Bischoff-Grethe, A., Dinges, D., Ayalon, L., Mednick, S.C., \& Meloy, M.J. (2005). The Neural Basis of the Psychomotor Vigilance Task. Sleep. 28 (9), 1059-1068. Tasks. PLoS ONE, 7(11).

Pontifex, M. B., \& Hillman, C. H. (2007). Neuroelectric and behavioral indices of interference control during acute cycling. Clinical Neurophysiology, 118(3), 570-580.

Jennings, J. R., \& Wood, C. C. (1976). The e-adjustment procedure for repeated- measures analyses of variance. Psychophysiology, 13, 277-278.

Lambourne K., \& Tomporowski PD. (2010). The effect of acute exercise on cognitive task performance: a meta-regression analysis. Brain Research Reviews, 1341, 12-24.

Langner, R., \& Eickhoff, S. B. (2013). Sustaining Attention to Simple Tasks: A Meta-Analytic Review of the Neural Mechanisms of Vigilant Attention. Psychological bulletin, 139(4), 870-900.

Lawrence, N. S., Ross, T. J., Hoffmann, R., Garavan, H., \& Stein, E. A. (2003). Multiple Neuronal Networks Mediate Sustained Attention. Journal of Cognitive Neuroscience, 15 (7), 10281038.

Luque-Casado, A., Perakakis, P., Ciria, L., \& Sanabria, D. (2016). Transient autonomic responses during sustained attention in high and low fit young adults. Scientific Reports. 6: 27556.

Luque-Casado, A., Perakakis, P., Hillman, C. H., Kao, S.C., Llorens, F., Guerra, P., \& Sanabria, D. (2015). Differences in Sustained Attention Capacity as a Function of Aerobic Fitness. Medicine and Science in Sports and Exercise. 48 (5), 887-895.

McMorris, T., Davranche, K., Jones, G., Hall, B., Corbett, J., \& Minter, C. (2009). Acute incremental exercise, performance of a central executive task, and sympathoadrenal system and hypothalamic-pituitary-adrenal axis activity. International Journal of Psychophysiology, 73(3), 334-340. 
McMorris, T., \& Hale, B.J. (2012). Differential effects of differing intensities of acute exercise on speed and accuracy of cognition: A meta-analytical investigation. Brain Cognition, 80 (3), $338-351$.

McMorris, T., \& Hale, B.J. (2015). Is there an acute exercise-induced physiological/biochemical threshold which triggers increased speed of cognitive functioning? A meta-analytic investigation. Journal of Sport and Health Science, 4, 4-13.

Muto, V., Jaspar, M., Meyer, C., Kussé, C., Chellappa, S. L., Degueldre, C., Balteau, E., Shaffii-Le Bourdiec, A., Luxen, A., Middleton, B., Archer, S.N., Phillips, C., Collette, F., Vandewalle, G., Dijk, D-J., \& Maquet, P. (2016). Local modulation of human brain responses by circadian rhythmicity and sleep debt. Science, 353(6300), 687-690.

Myers, J., \& Ashley, E. (1997). Dangerous curves. A perspective on exercise, lactate, and the anaerobic threshold. Chest, 111, 787-795.

Oken, B. S., Salinsky, M. C., \& Elsas, S. M. (2006). Vigilance, alertness, or sustained attention: physiological basis and measurement. Clinical Neurophysiology, 117(9), 1885-1901.

Pesce, C., Capranica, L., Tesittore, A., \& Figura, F. (2002). Effects of a submaximal physical load on the orienting and focusing of visual attention. Journal of Human Movement Studies, 42, 401-420.

Petersen, S. E., \& Posner, M. I. (2012). The Attention System of the Human Brain: 20 Years After. Annual review of neuroscience, 35, 73-89.

Rosenberg, M. D., Finn, E. S., Scheinost, D., Papademetris, X., Shen, X., Constable, R. T., \& Chun, M. M. (2016). A neuromarker of sustained attention from whole-brain functional connectivity. Nature neuroscience, 19(1), 165-171.

Sarter, M., Given, B., \& Bruno, J.P. (2001). The cognitive neuroscience of sustained attention: where top-down meets bottom-up. Brain Research Reviews, 35(2):146-160.

Tanaka, H., Monahan, K. D. \& Seals, D. R. (2001). Age-predicted maximal heart rate revisited. Journal of American College of Cardiology, 37, 153-156. 
391 Voss, M. W., Nagamatsu, L. S., Liu-Ambrose, T., \& Kramer, A. F. (2011). Exercise, brain, and 392 cognition across the life span. Journal of Applied Physiology, 111(5), 1505-1513.

393 Wagenmakers, E.-J., Wetzels, R., Borsboom, D., van der Maas, H. L. J., \& Kievit, R. A. (2012). An Agenda for Purely Confirmatory Research. Perspectives on Psychological Science: A Journal of the Association for Psychological Science, 7(6), 632-638.

396 Walsh, V. (2014). Is sport the brain’s biggest challenge? Current Biology, 24, 859-860.

397 Yeh, M.P., Gardner, R.M., Adams, T.D., Yanowitz, F.G., \& Crapo, R.O. (1983) “Anaerobic threshold": problems of determination and validation. Journal of Applied Physiology, 55, $1178-1186$

400 Yerkes, R.M. \& Dodson, J.D. (1908). The relation of strength of stimulus to rapidity of habitformation. Journal of Comparative Neurology and Psychology, 18, 459-482. 
Table 1. Anthropometrical and physiological characteristics in Experiments 1 and 2 (mean \pm SD).

\section{Experiment 1 Experiment 2}

\begin{tabular}{lll}
\hline Sample Size & 24 & 18 \\
Gender & 12 females & 18 females \\
Age (yrs) & $20.29 \pm 0.95$ & $19.94 \pm 1.98$ \\
Height (cm) & $170.92 \pm 9.34$ & $163.33 \pm 2.85$ \\
Weight (kg) & $66.70 \pm 8.91$ & $61.32 \pm 6.31$ \\
Body fat (\%) & $22.72 \pm 1.54$ & $23.12 \pm 2.61$ \\
\hline Power max (W) & $173.75 \pm 40.03$ & $96.67 \pm 21.49$ \\
Relative power (W/kg) & $2.64 \pm 0.48$ & $1.58 \pm 0.33$ \\
VAT (ml/kg/min) & $34.26 \pm 6.32$ & $23.72 \pm 5.61$ \\
\hline
\end{tabular}

403

Table 2. Physiological parameters in Experiments 1 and 2 (mean $\pm \mathrm{SD}$ ).

\begin{tabular}{|c|c|c|c|c|}
\hline \multicolumn{5}{|l|}{ Experiment 1} \\
\hline Effort condition & HR (bpm) & \% of HR max & $\%$ of VAT & RPE \\
\hline \multicolumn{5}{|l|}{$40 \%$ of VAT } \\
\hline $\begin{array}{l}\text { Very light }<50 \% \text { of } \mathrm{HR} \text { max } \\
\mathbf{6 0 \%} \text { of VAT }\end{array}$ & $79 \pm 9$ & $40.77 \pm 4.51$ & $49.53 \pm 5.69$ & $6.75 \pm 0.74$ \\
\hline $\begin{array}{l}\text { Light } 50-<64 \% \text { of HR max } \\
\mathbf{8 0 \%} \text { of VAT }\end{array}$ & $97 \pm 5$ & $50.05 \pm 2.76$ & $60.45 \pm 1.86$ & $8.67 \pm 1.01$ \\
\hline $\begin{array}{l}\text { Moderate } 64-<77 \% \text { of } \mathrm{HR} \\
\max \end{array}$ & $129 \pm 7$ & $66.57 \pm 3.82$ & $80.27 \pm 3.48$ & $12.17 \pm 1.09$ \\
\hline $\begin{array}{l}\mathbf{1 0 0} \% \text { of VAT } \\
\text { Vigorous } 85-<100 \% \text { of HR } \\
\text { max }\end{array}$ & $159 \pm 12$ & $82.05 \pm 6.23$ & $99.07 \pm 6.64$ & $16.92 \pm 1.35$ \\
\hline \multicolumn{5}{|l|}{ Experiment 2} \\
\hline Effort condition & HR (bpm) & $\%$ of HR max & $\%$ of VAT & RPE \\
\hline $\begin{array}{l}\text { Low-effort condition } \\
\text { Low load } \\
\text { Light-moderate-effort } \\
\text { condition }\end{array}$ & $84 \pm 7$ & $43.97 \pm 1.45$ & $52.13 \pm 4.20$ & $8.83 \pm 2.07$ \\
\hline $75 \%$ of VAT & $120 \pm 11$ & $63.04 \pm 0.80$ & $74.25 \pm 6.97$ & $12.39 \pm 1.65$ \\
\hline
\end{tabular}


Figure 1

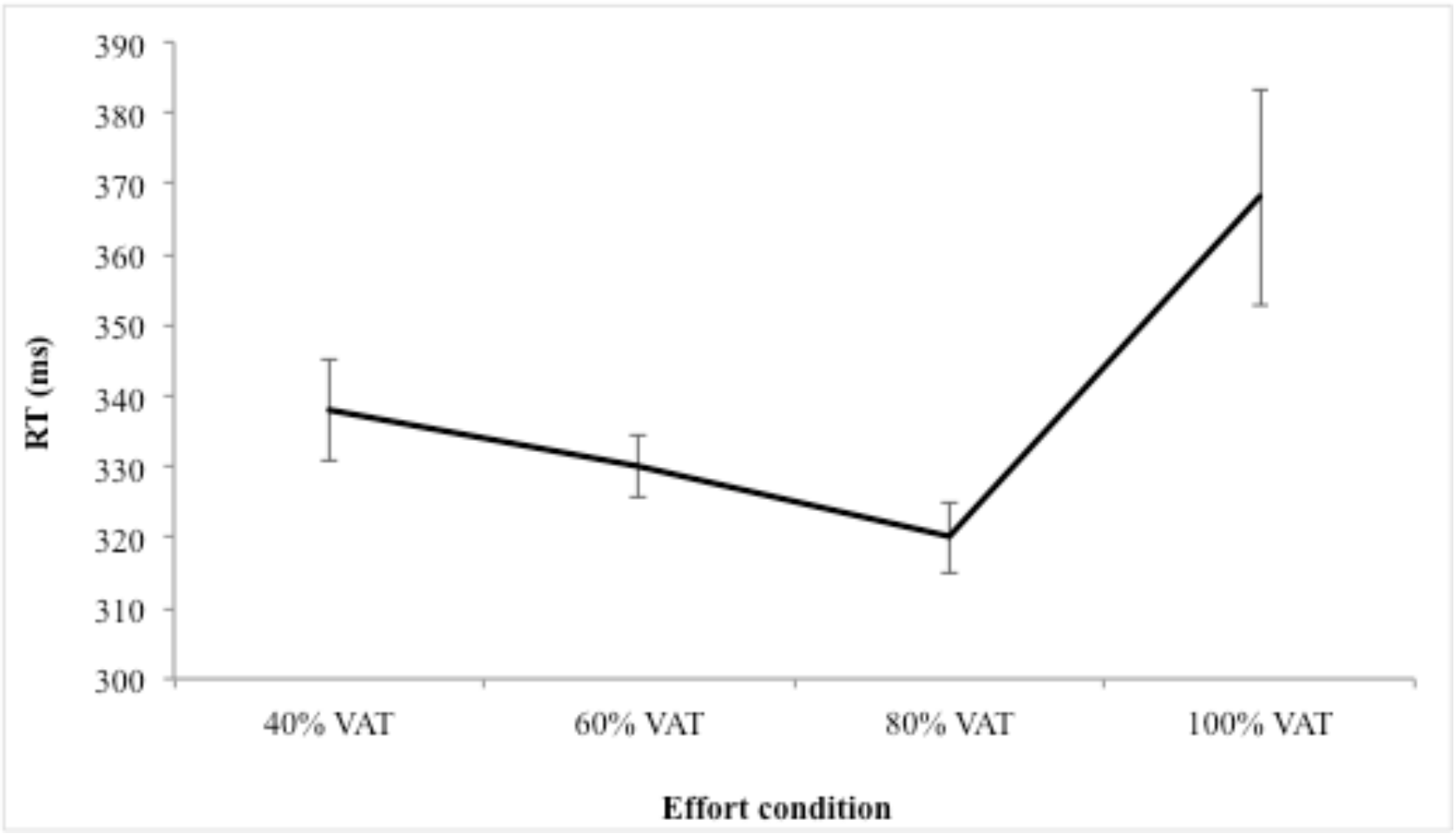

407

408 Figure 2.

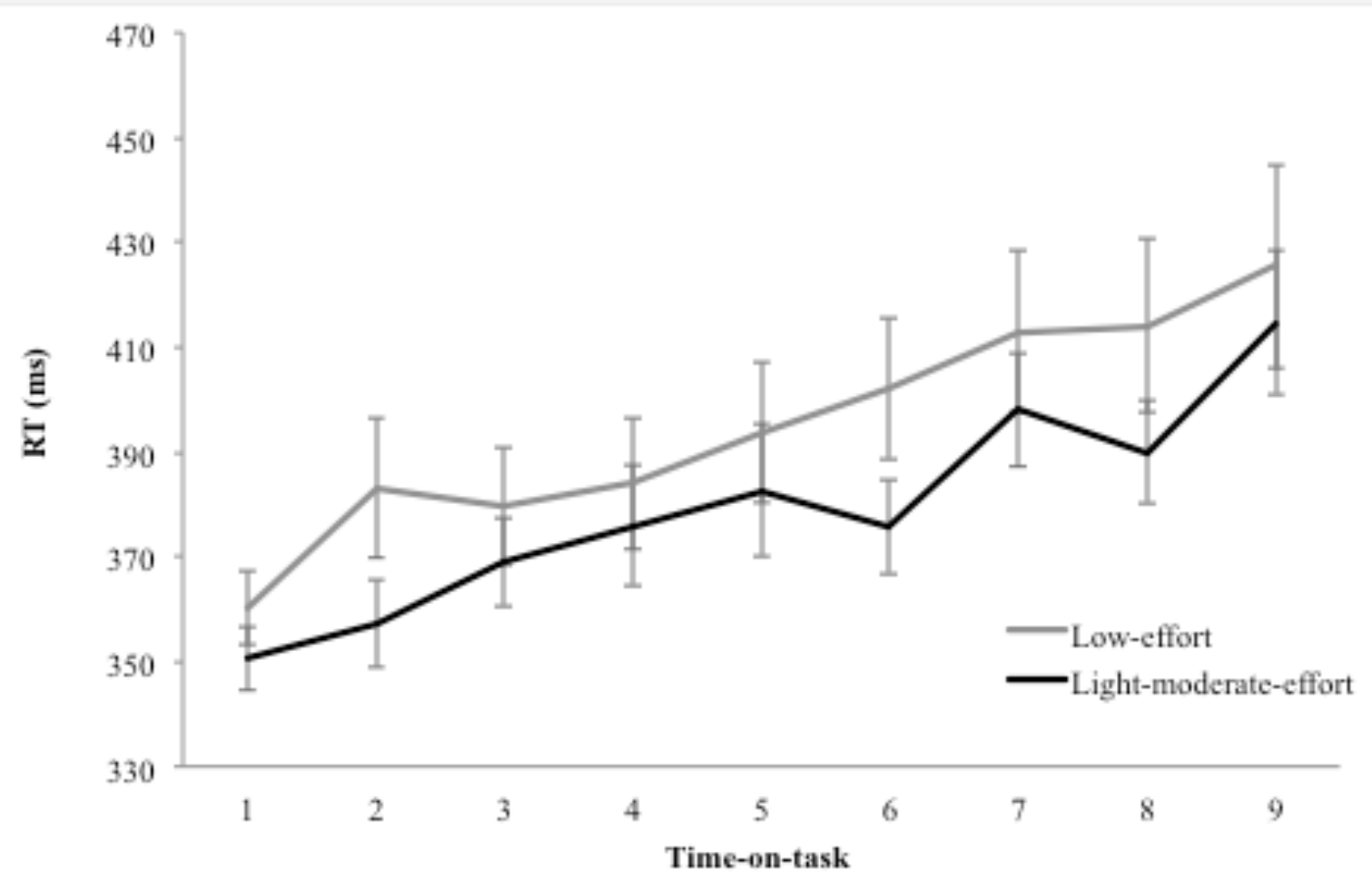

409

410 


\section{Captions.}

414 Figure 1. Mean RT $( \pm \mathrm{SE})$ as a function of effort condition in Experiment 1.

415 Figure 2. Participants' mean RT as a function of effort condition and time-on-task in Experiment 2.

416

417

418

419

420

421

422

423

424

425

426

427 